\title{
Estudio Teórico de las Propiedades Electroconductoras de Oligómeros del Compuesto 4H-[1,2,4]-Triazol
}

\author{
Walter J. Cuadro, Adolfo E. Ensuncho y Jesús M. López \\ Universidad de Córdoba, Departamento de Química, Grupo de Química Computacional, Cra 6 № 76-103, \\ Córdoba-Colombia (e-mail:wjcuadro@gmail.com; adem9abril@hotmail.com; \\ jmanuellopez.gqc@gmail.com)
}

Recibido Nov. 15, 2012; Aceptado Ene. 07, 2013; Versión final recibida Mar. 06, 2013

\begin{abstract}
Resumen
Se estudiaron computacionalmente una serie de oligómeros de 2 a 30 anillos del compuesto $4 \mathrm{H}-[1,2,4]-$ Triazol al nivel B3LYP/6-31G(d), con el fin de evaluar sus propiedades electro conductoras y contribuir con el diseño de nuevos materiales poliméricos transportadores de electrones (dopado $n$ ). La brecha de energía prohibida predicha para el polímero mediante extrapolación binomial de segundo orden, mostró buen acuerdo con los reportes experimentales. Asimismo, los cálculos teóricos mostraron que los procesos de dopado mejoraron notablemente la conducción de los diferentes oligómeros y polímeros $(1 / n=0)$, obteniéndose brechas de energía prohibida de $0.095 \mathrm{eV}$ para el dopado $\mathrm{n}$ y $0.017 \mathrm{eV}$ para el dopado $\mathrm{p}$ respectivamente. Además, se encontró que las distancias de enlace $\mathrm{C}-\mathrm{N}, \mathrm{N}-\mathrm{N}$ y $\mathrm{C}-\mathrm{C}$, son fuertemente distorsionadas en los oligómeros cargados, implicando con ello la pérdida parcial de la aromaticidad en el anillo triazólico, probablemente por la formación de estructuras quinoides.
\end{abstract}

\section{Theoretical Study of The Electroconductive Properties of Oligomers $4 \mathrm{H}-[1,2,4]-T r i a z o l e$}

\begin{abstract}
A series of oligomers of 2 to 30 rings of the compound $4 \mathrm{H}-[1,2,4]$-triazole, were computationally studied at level 6-31G(d) in order to evaluate their electro-conductive properties and contribute to design of novel electron carrier polymers (n-type). The band-gap predicted for polymer by extrapolation approach adopted a binomial-fitting scheme of second order, showed good agreement with experimental data. Also, the theoretical calculations show that doping processes improved the conduction in oligomers and polymers $(1 / n=0)$, obtaining band-gap of $0.095 \mathrm{eV}$ for $n$-type and $0.017 \mathrm{eV}$ for $p$-type respectively. It was found that the $\mathrm{C}-\mathrm{N}, \mathrm{N}-\mathrm{N}$ and $\mathrm{C}-\mathrm{C}$ bond lengths in the oligomers are strongly distorted in the charged state, implying the partial loss of aromaticity on triazole ring, probably caused by the formation of quinoid-like structures.
\end{abstract}

Keywords: conducting polymers, oligomers, triazole, band-gap, quinoid-like structures 


\section{INTRODUCCIÓN}

Después del descubrimiento de Shirakawa (MacDiarmid, 2001; Shirakawa et al., 1977), la ciencia de los polímeros conductores ha cautivado la atención de muchos investigadores a nivel multidisciplinar, involucrando áreas como la física, química, electrónica y biomedicina (Casanovas et al., 2005). Este notable interés de se debe en parte, al considerable número de aplicaciones de estos materiales en la fabricación de dispositivos ópticos, diodos emisores de luz (LEDs), sensores químicos y músculos artificiales (Bar-Cohen, 2007; Liu et al., 2011; Myers y Xue, 2012). De ahí que, la síntesis de nuevos polímeros $\pi-$ conjugados sea esencial para mejorar las propiedades ópticas y electroconductoras de estos materiales (Vivas-Reyes, 2008), así como también, su estabilidad medioambiental (Casanovas et al., 2005).

Una característica importante de los polímeros es su fácil procesabilidad, lo cual ofrece la ventaja de producir dispositivos electrónicos a bajo costo en relación con los materiales conductores tradicionalmente usados (Heeger, 2001). Por ello, se han realizado estudios tanto teóricos como experimentales de un gran número de polímeros $\pi$-conjugados con estructuras químicas diversas de los cuales los más representativos son los basados en unidades heterocíclicas del pirrol (Salmón et al., 1982; Zhu et al., 2006) y tiofeno (Kertesz et al., 2009; Liu et al., 2011), debido a su alta conductividad luego de ser sometidos a un proceso de dopado (MacDiarmid et al., 1985).

Otra unidad estructural importante para el diseño de polímeros y copolímeros con propiedades electroconductoras, corresponde al compuesto heterocíclico $4 \mathrm{H}$-[1,2,4]-triazol designado como TAZ, ya que ofrece la posibilidad de generar diodos emisores de luz (OLEDs, por sus siglas en inglés) (Kido et al., 1993). Recientemente, Janietz y colaboradores (Janietz et al., 2004) reportaron la síntesis de un nuevo polímero basado en unidades de 4H-[1,2,4]-triazol, como un candidato potencial en el diseño de materiales poliméricos, que permitan el transporte de electrones en dispositivos electrónicos. Esta última característica es indispensable cuando se desea obtener polímeros semiconductores cuyos portadores de carga sean esencialmente electrones.

Por otro lado, agregar unidades TAZ en la síntesis de copolímeros basados en 10-hexilfenotiazina favorece las propiedades electroconductoras de la cadena polimérica (Choi et al., 2009). Este hecho resalta que los compuestos TAZ, son piezas clave en la funcionalización de estructuras poliméricas (Shin et al., 2011). Así por ejemplo, se ha reportado la síntesis y caracterización de polímeros energéticos conteniendo triazoles (Shin et al., 2011).

La funcionalización con TAZ, da al polímero energético cierta ventaja con respecto a los materiales comúnmente usados en formulaciones propelentes y que en su mayoría corresponden a polímeros del tipo azido glicidilo (GAPs, por sus siglas en inglés) (Nazare et al., 1992; Wang et al., 2007). Esta ventaja adicional consiste en generar un polímero aglutinador en la formulación propelente, pero que sea más energético que GAP y ecológicamente amigable. Cabe resaltar, que esta última característica es uno de los objetivos que a futuro se espera alcanzar en el desarrollo de polímeros $\pi$-conjugados (Casanovas et al., 2005), dado que los dispositivos conductores basados en metales inorgánicos son tóxicos y afectan algunos ciclos biológicos indispensables para el sustento de la vida (Haye et al., 2007, Nahmani et al., 2007).

Los polímeros, también se han investigado mediante técnicas de modelización molecular, hasta el punto que se ha convertido en una herramienta clave para el diseño de nuevos polímeros semiconductores, ya que ofrece la posibilidad de predecir la estructura química y propiedades electroconductoras de un material sin que este haya sido sintetizado previamente (Curcó y Alemán, 2007; Derosa, 2009; Hosoya et al., 1987; Satyanarayana et al., 2010). La literatura científica revela que las familias de polímeros que generalmente se estudian mediante técnicas de modelización, corresponden a tiofenos y pirroles respectivamente (Curcó y Alemán, 2007; Raos, 2003; Ripoll et al., 2010; Rodríguez-Ropero et al., 2008, Shaktawat et al., 2007). Sin embargo, para los polímeros heterocíclicos TAZ, los reportes son escasos (Jansson et al., 2006; Li et al., 2011).

En razón a lo anterior, y dada la importancia de los triazoles como bloques de construcción en arquitecturas poliméricas, se propone el estudio computacional de oligómeros de 2 a 30 anillos del compuesto $4 \mathrm{H}-[1,2,4]$ Triazol, que aun no han sido sintetizados, con el fin de evaluar sus propiedades electro-conductoras y potencialidad como material para el transporte de electrones. Además, se reportan los dopados tipo $p$ y $n$ para estos oligómeros. 


\section{METODOLOGÍA}

\section{Detalles Computacionales}

Los cálculos computacionales para los oligómeros de 2 a 30 anillos del compuesto 4H-[1,2,4]-Triazol, designados como Taz- $\mathrm{H}_{(2-30)}$, el subíndice indica el numero de anillos, se desarrollaron a través del paquete computacional Gaussian03 (rev. E.01) (Frisch, 2004). Los oligómeros $\mathrm{Taz}_{-} \mathrm{H}_{(2-30)}$ se optimizaron sin restricciones a la geometría con el modelo químico B3LYP/6-31G(d) (Vivas-Reyes et al., 2008), tomando como geometría de partida una conformación anti-planar, donde los ángulos diedros entre los anillos adyacentes son de $180^{\circ}$. De igual manera, para investigar la modificación estructural inducida por los dopados tipo p y $\mathrm{n}$, los oligómeros fueron optimizados al mismo nivel de teoría. Las propiedades de conducción de los polímeros se calcularon con base en las propiedades eléctricas, energía de ionización (EI) y afinidad electrónica (AE), teniendo en cuenta la diferencia energética entre los oligómeros neutros y los oligómeros cargados (ecuaciones 1 y 2). Para la estimación de la brecha de energía prohibida o bandgap $(\mathrm{Eg})$, se tomó la diferencia entre el orbital molecular ocupado de más alta energía (HOMO) y el orbital molecular desocupado de más baja energía (LUMO), haciendo uso de la ecuación 3 (Vivas-Reyes et al., 2008).

$$
\begin{aligned}
& E I=E_{\text {catión }}-E_{\text {neutro }} \\
& A E=E_{\text {anión }}-E_{\text {neutro }} \\
& E_{g}=E_{\text {LUMO }}-E_{\text {HOMO }}
\end{aligned}
$$

En este trabajo se empleó la aproximación de extrapolación para obtener propiedades electrónicas del polímero a cadena de longitud infinita (Wang et al., 2004). Convencionalmente, se adopta un esquema de ajuste lineal para efectuar dicha extrapolación. Sin embargo, en este trabajo se encontró que para los oligómeros de triazol estudiados, se obtuvo un mejor resultado a través de ajuste binomial, el cual típicamente suele definirse mediante la ecuación general: $Y=a+b X+c X^{2}$, donde $X$ es igual a $1 / n$ (Liu et al., 2006). El término $1 / \mathrm{n}$, es el recíproco del número de unidades repetidas TAZ y $Y$, corresponde al valor de los parámetros correlativos a la estructura electrónica; tales como, $\mathrm{El}, \mathrm{AE}$ y $\mathrm{E}_{\mathrm{g}}$ respectivamente. Este procedimiento de extrapolación se aplicó tanto para el polímero neutro como los polímeros con dopado $\mathrm{n}$ y p respectivamente.

Cabe resaltar que, aunque las cadenas poliméricas propuestas en este trabajo no se construyeron a partir de los tautómeros TAZ más abundantes, es decir, las especies 1H y 2H (Bele y Singhvi, 2011), la elección del tautómero minoritario $4 \mathrm{H}$ se hizo teniendo en cuenta que dicho tautómero es un candidato potencial para la construcción de materiales transportadores de electrones, como fue reportado por Janietz y colaboradores (Janietz et al., 2004), mediante la síntesis de un nuevo polímero basado en unidades de $4 \mathrm{H}$ $[1,2,4]$-triazol.

\section{RESULTADOS Y DISCUSIÓN}

Como se muestra en la figura 1, la geometría del dímero de triazol predicha al nivel B3LYP/6-31G(d), no presentó distorsión con respecto a la configuración anti-planar $\left(\sim 180^{\circ}\right)$. Esta tendencia se mantuvo para el resto de oligómeros TAZ.<smiles>c1nnc(-c2nnc[nH]2)[nH]1</smiles>

Fig. 1: Ángulo torsional del dímeroTAZ en conformación anti-planar.

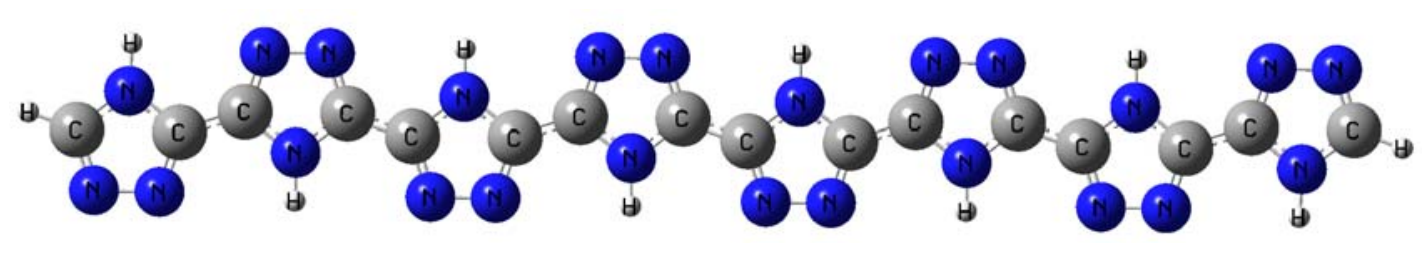

Fig. 2: Geometría optimizada al nivel B3LYP/6-31G(d) del oligómero TAZ-H ${ }_{(8)}$ 
En la figura 2, se presenta la geometría optimizada para el oligómero TAZ de 8 anillos, siendo evidente que una característica estructural de estos oligómeros es su elevada planaridad molecular, de modo que la conducción se ve favorecida, ya que hay un mayor solapamiento de los orbitales $p$ o conjugación m, de tal manera que emergen bandas dadoras de alta energía y bandas aceptoras de baja energía (Casanovas et al., 2005). En la tabla 1, se presentan los valores de las propiedades eléctricas calculadas para los oligómeros TAZ en estados neutro y dicatiónicos (dopados $n$ y $p$ ) respectivamente. De acuerdo con estos valores, se observa que la energía de ionización de los oligómeros disminuye conforme aumenta el número de anillos de triazol, manteniéndose la tendencia hasta llegar al polímero $\left(T A Z_{(\infty)}\right)$. Este resultado indica lo fácilmente oxidables que son estos sistemas, dado que facilitan la salida de electrones y por ende un dopado tipo $\mathrm{p}$ para este material se favorece. Asimismo, la afinidad electrónica muestra una marcada tendencia de estos sistemas a retener electrones conforme incrementa el número de anillos de triazol, de tal manera que pueden ser fácilmente reducidos y por lo tanto el dopado tipo $n$ también se favorece. El resultado obtenido para la afinidad electrónica de estos sistemas resulta interesante, puesto que para el diseño materiales poliméricos transportadores de electrones, se debe tener en cuenta que posean propiedades aceptoras de electrones o expresado en otros términos; una elevada afinidad electrónica.

También, con base en la afinidad electrónica predicha para los oligómeros y el polímero se puede afirmar que estos materiales son estables frente a la acción de oxígeno molecular, dado que mediante la $\mathrm{AE}$, se tiene una idea de la estabilidad del anión-radical frente a oxígeno. Recientemente, Risko y colaboradores (Risko et al., 2005) reportaron la afinidad electrónica predicha para oxígeno al nivel B3LYP, obteniéndose un valor de $-0.59 \mathrm{eV}$. De modo que, la $\mathrm{AE}$ predicha para $\mathrm{TAZ} \mathrm{Z}_{(\infty)}$, es significativamente mayor y por ende, se presume que estos materiales presentarían una estabilidad alta frente a oxígeno molecular. De lo anterior es claro que, estos materiales poliméricos basados en unidades TAZ, son potenciales candidatos para el diseño de OLEDs, dado que los materiales transportadores de huecos tradicionales usados para este fin, requieren el uso de cátodos con funciones de trabajo bajas, de tal manera que la eficiencia electroluminiscente aumente (Jansson et al., 2006). Sin embargo, el uso de estos cátodos con funciones de trabajo bajas, limita el tiempo de vida útil del material, pues son susceptibles al oxígeno molecular del medio. Por lo tanto, con base en los resultados predichos, es factible suponer que un polímero basado en TAZ al ser más estable frente a oxígeno molecular supera los inconvenientes ya mencionados en dispositivos OLEDs.

Tabla 1: Energía de ionización, afinidad electrónica y energía band-gap para los oligómeros de TAZ al nivel B3LYP/6-31G(d). Las unidades están expresadas en eV

\begin{tabular}{|c|c|c|c|c|c|}
\hline \multirow[t]{2}{*}{ Oligómero } & \multicolumn{3}{|c|}{ Band-gap HOMO-LUMO $\left(\mathrm{E}_{\mathrm{g}}\right)$} & \multirow[t]{2}{*}{$\mathrm{AE}$} & \multirow[t]{2}{*}{ El } \\
\hline & -2 & 0 & 2 & & \\
\hline $\mathrm{TAZ}_{(4)}$ & 1.475 & 4.679 & 1.492 & -0.318 & 8.053 \\
\hline $\operatorname{TAZ}_{(6)}$ & 0.610 & 4.385 & 0.724 & -0.830 & 7.720 \\
\hline $\operatorname{TAZ}_{(8)}$ & 0.365 & 4.261 & 0.413 & -1.126 & 7.524 \\
\hline $\mathrm{TAZ}_{(10)}$ & 0.234 & 4.196 & 0.254 & -1.321 & 7.387 \\
\hline $\mathrm{TAZ}_{(15)}$ & 0.139 & 4.127 & 0.144 & -1.604 & 7.178 \\
\hline $\mathrm{TAZ}_{(20)}$ & 0.099 & 4.102 & 0.100 & -1.769 & 6.937 \\
\hline $\operatorname{TAZ}_{(30)}$ & 0.067 & 4.085 & 0.061 & -1.968 & 6.759 \\
\hline $\mathrm{TAZ}(\infty)$ & 0.095 & 4.039 & 0.017 & -2.294 & 6.454 \\
\hline
\end{tabular}

De igual manera, en la tabla 1, se presentan los valores predichos para la brecha de energía prohibida o gap HOMO-LUMO, de los oligómeros y polímero respectivamente. Para mejorar la predicción del Eg, fue esencial el uso de oligómeros con tamaño superior a 20 monómeros, lo cual estuvo en buen acuerdo con lo previamente reportado por Zade y Bendikov (Zade y Bendicov, 2006), quienes indicaron que la utilización de oligómeros conformados por diez unidades monoméricas, no es suficiente para predecir el band gap y que para cadenas oligoméricas con $n>12$, es preferible usar un ajuste binomial de segundo orden.

El band-gap calculado para los estados neutros, dicatión (dopado tipo p) y dianión (dopado tipo n) de los oligómeros $\mathrm{TAZ}_{(4-30)}$, disminuye con el aumento del numero monómeros en la cadena, lo que está en total acuerdo con la teoría de bandas. Desde el punto de vista de esta teoría, la oxidación y reducción (dopado $p$ y $\mathrm{n}$ respectivamente), origina la aparición de estados electrónicos en el band-gap del material, que influyen notablemente en el proceso de conducción ( MacDiarmid, 2001). De ahí que, en la tabla 1, se observe que los oligómeros dopados y el polímero, muestran valores significativamente bajos de band-gap con respecto a los sistemas no dopados (neutros).

Este hecho se explica, considerando que la presencia de estados dicatiónicos y dianiónicos, deja libres algunos electrones en los oligotriazoles, de modo que, estos pueden abandonar la banda de valencia y alcanzar la banda de conducción, mediante una transición electrónica que requiere una energía menor a la energía del band-gap. 


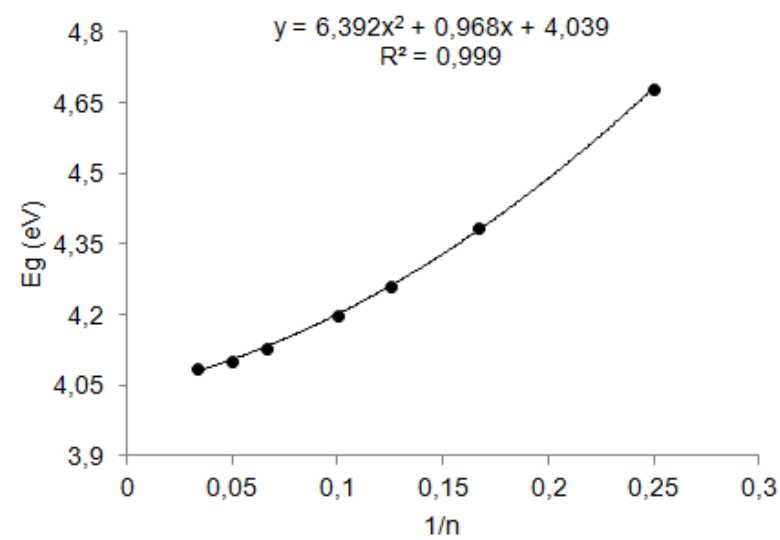

Fig. 3: Extrapolación binomial de segundo orden para $T A Z_{(4-30)}$ sin dopado

En las figuras 3 y 4 , se muestran las tendencias obtenidas bajo los esquemas de ajuste binomial de segundo orden para los oligómeros estudiados en sus estados no dopado y dopado respectivamente.

A

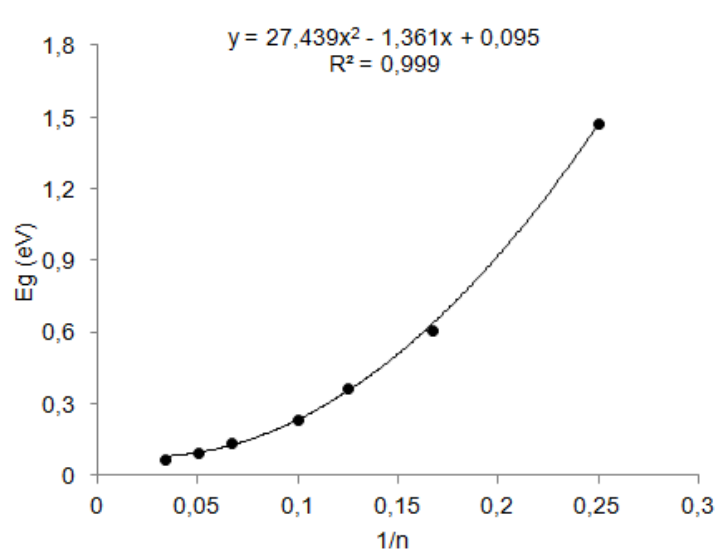

B

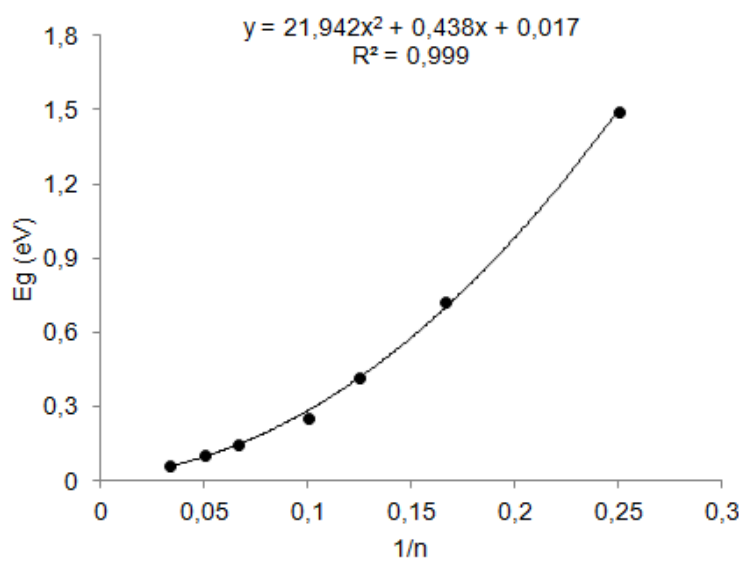

Fig. 4: Extrapolación binomial de segundo orden para $T A Z_{(4-30)}$ bajo los dopados tipo $n(A)$ y tipo $p(B)$ respectivamente

Para estos sistemas, el ajuste binomial de segundo orden mostró una buena correlación entre el gap HOMO-LUMO y el inverso del número de unidades triazólicas $(1 / n)$. De acuerdo, con las predicciones derivadas de la ecuación cuadrática ( $n=30$ y $R^{2}=0.999$, en todos los casos), se estimaron los valores de band-gap, extrapolando a cadena de longitud infinita, es decir considerando $(1 / n=0$, si $n=\infty)$, del polímero neutro, dicatiónico y dianiónico respectivamente. Se observa que para el polímero neutro se obtuvo un Eg de $4.039 \mathrm{eV}$ (tabla 1). Este band-gap predicho, mostró concordancia con el band-gap óptico reportado recientemente por Janietz y colaboradores (Janietz et al., 2004) para el polímero poly(4-hexil-1,2,4-triazol$4 \mathrm{H}$ ), el cual tiene una estructura química similar a los polímeros propuestos en este estudio, confirmando que el nivel de teoría usado fue adecuado para la caracterización de las propiedades electrónicas de estos oligómeros. Asimismo, se observa que el polímero sin dopar no conduce la corriente eléctrica, ya que presenta un valor de band-gap superior a $2 \mathrm{eV}$ (Casanovas et al., 2005).

Además, como era de esperarse la extrapolación a polímero para los dopados $n$ y $p$, mostró que la conducción incrementa significativamente, dado que los gap HOMO-LUMO son $0.095 \mathrm{eV}$ y $0.017 \mathrm{eV}$ respectivamente. Este resultado deja en evidencia que la deslocalización en los anillos del triazol, no es suficiente para que el material se comporte como un conductor, sino que también es necesario que el material sea dopado. Se observa también, que el dopado tipo $p$, le confiere al polímero una mayor electroconducción que el dopado $n$. No obstante, a partir del valor de band-gap predicho para el dopado $\mathrm{n}$ (tabla 1), se presume que estos polímeros se comporten como materiales transportadores de electrones y aunque no hay información experimental suficiente para soportar esta afirmación, los resultados arrojados por los cálculos computacionales son razonables y consistentes.

Ahora bien, es un hecho experimental conocido que el desarrollo de materiales transportadores de huecos (dopado tipo $\mathrm{p}$ ), ha sido superior al desarrollo de materiales para el transporte de electrones (Hughes y Bryce, 2005). Este desarrollo poco equilibrado, trae consigo que en los dispositivos emisores de luz 
actuales, la zona de recombinación se encuentre desplazada hacia el cátodo y por ende disminuya la eficiencia electroluminiscente, debido a la extinción de excitones por el cátodo metálico (Hughes y Bryce, 2005). De modo que, se requiere de la síntesis de polímeros que permitan el transporte no solo de huecos sino también de electrones. En este contexto, los polímeros basados en TAZ son buenos candidatos, ya que, de los valores de band-gap predichos mediante la extrapolación a polímero (tabla 1), es evidente que los dopados $n$ y $\mathrm{p}$, podrían establecer un balance razonable en la inyección de carga a partir de los electrodos, así como también, un transporte eficiente de huecos y electrones, siendo por lo tanto plausible la utilización de este material como una capa orgánica luminiscente en un dispositivo OLED.

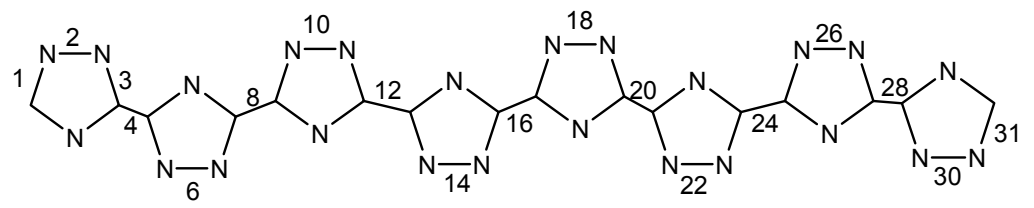

Fig. 5: Longitudes de enlace, carbono-nitrógeno, carbono-carbono y nitrógeno-nitrógeno en el octámero de triazol

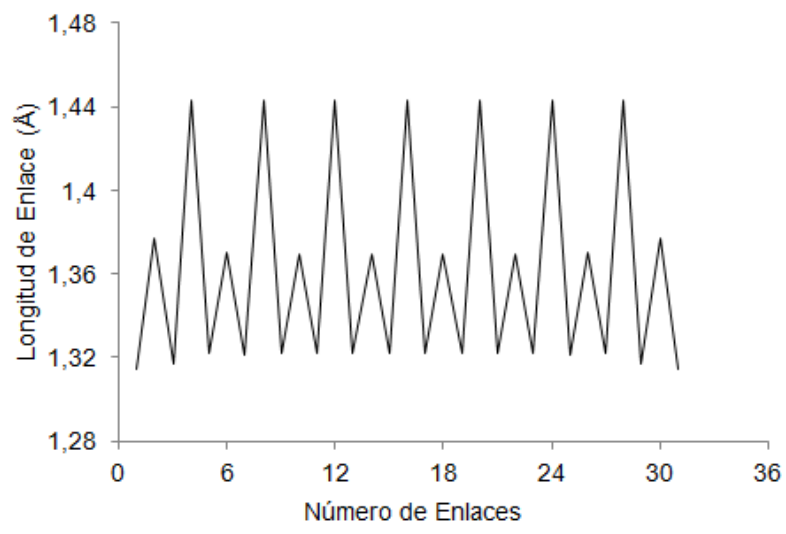

$6 a$

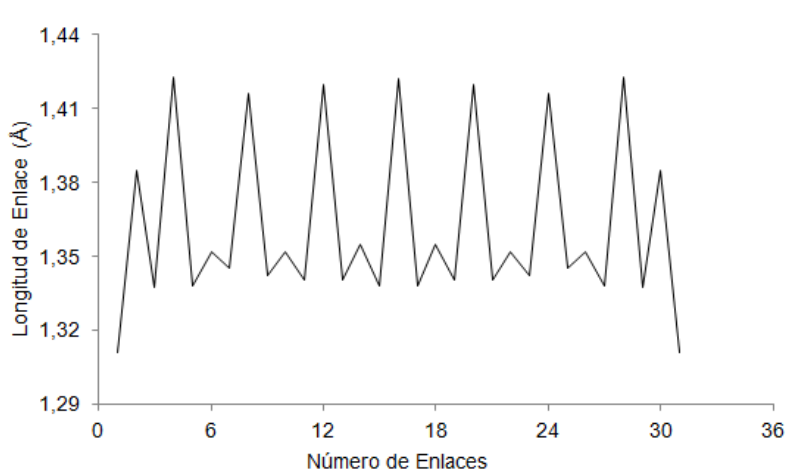

$6 b$

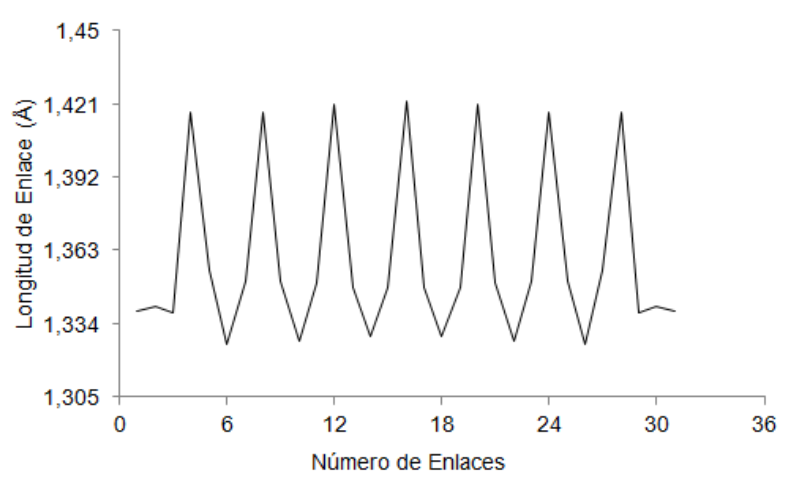

$6 \mathrm{c}$

Fig. 6: Longitudes de enlace C-N, C-C y N-N optimizadas al nivel B3LYP/6-31G(d), para el octámero neutro (6a), dianiónico (6b) y dicatiónico (6c)

Con respecto a los procesos de dopado, se espera que sobre los oligómeros ocurra una alteración tanto en su estructura electrónica como en su geometría. Para comprobar este efecto se compararon los parámetros geométricos predichos para el oligómero de ocho anillos en sus estados neutro y diónicos. En la figura 5, se presentan las distancias de enlace consideradas para llevar a cabo el análisis. En este análisis se encontró que las distancias $\mathrm{C}-\mathrm{N}, \mathrm{C}-\mathrm{C}$ y N-N , en el oligómero de ocho anillos se encuentran distorsionadas en los estados diónicos. Estas distorsiones se observan claramente en la figura 6, donde el oligómero neutro no presenta longitudes de enlace equivalentes con respecto a los octámeros cargados, lo cual es debido a la resonancia en el anillo triazólico. De modo que, la geometría más estable para este oligómero y en general para el resto de oligómeros corresponde a una estructura aromática que se extiende a lo largo de la cadena.

Este comportamiento varía en las figuras $6 \mathrm{~b}$ y $6 \mathrm{c}$ respectivamente, donde la resonancia se pierde en gran medida. En consecuencia, la pérdida de la aromaticidad induce acortamientos o elongaciones en las longitudes de enlace, lo cual probablemente se debe a la formación de estructuras quinoides (Alemán et al., 2005; Brédas et al., 1984). De ahí que, en la figura $6 \mathrm{~b}$ la transferencia de carga inducida por el dopado tipo 
n (agente dopante donador de electrones), induce fuertes distorsiones sobre la cadena oligomérica. Asimismo, para la especie dicatiónica también se observa esta misma tendencia de distorsión de la geometría. Un aspecto importante de la formación de estructuras quinoides, es que estas favorecen la conducción dentro de una cadena polimérica. En vista que la estructura $6 \mathrm{c}$, muestra un carácter quinoideo mayor que $6 \mathrm{~b}$; este resultado es consecuente con la alta conductividad mostrada por el polímero, cuando fue simulado bajo condiciones de dopado tipo $p$.

\section{CONCLUSIONES}

Los diferentes oligómeros TAZ, presentaron estructuras muy planas y valores de band gap menores a $2 \mathrm{eV}$, para los estados diónicos, sugiriendo con ello buenas propiedades electroconductoras para estos sistemas. Asimismo, las energías de ionización bajas y altas afinidades electrónicas, indicaron que los procesos de dopado $\mathrm{n}$ y $\mathrm{p}$ son favorecidos. Los resultados expuestos en este artículo, demuestran que las técnicas teóricas de modelización molecular basados en funcionales de la densidad, son adecuadas para evaluar las propiedades eléctricas de estos materiales, a la vez que permiten comprender mejor el fenómeno de la conducción eléctrica. Como resultado de los proceso de dopado, se observó la formación de estructuras quinoides, las cuales favorecieron notablemente la conductividad dentro de la estructura oligomérica. Las variaciones energéticas entre los oligómeros al extenderse la cadena hasta el infinito, mantuvieron las tendencias en las medidas de las propiedades electrónicas, haciendo posible predecir algunos parámetros importantes de las propiedades electroconductoras del polímero. Para este grupo de oligómeros, la extrapolación a polímero presentó mejores resultados adoptando un esquema de ajuste binomial en lugar del ajuste lineal tradicional. Las propiedades electroconductoras predichas para estos sistemas sugieren su potencialidad como materiales transportadores de electrones.

\section{AGRADECIMIENTOS}

Los autores de este artículo muestran su agradecimiento al Centro de Investigaciones de la Universidad de Córdoba (CIUC), por el soporte económico brindado para el desarrollo del estudio en el marco del proyecto de investigación código 1.2.08.109 y numeral FCB-03-09.

\section{REFERENCIAS}

Alemán, C. y otros cinco autores, Structural and electronic properties of 3,4-ethylenedioxythiophene, 3,4ethylenedisulfanylfurane and thiophene oligomers: A theoretical investigation, Synthetic Met: 149(2-3), 151156 (2005).

Bar-Cohen, Y, Artificial muscles based on electroactive polymers as an enabling tool in biomimetics, Proc. Inst. Mech. Eng. Pt. C. J. Mechan: 221(10), 1149-1156 (2007).

Brédas, J. L. y otros cuatro autores, Highly conducting polyparaphenylene, polypyrrole, and polythiophene chains: An ab initio study of the geometry and electronic-structure modifications upon doping, Phys. Rev. B: 29(12), 6761-6773 (1984).

Bele, D.S. y I. Sinhvi, A Review on 1, 2, 4-triazoles, AJBPAD: 1, 88-101 (2011).

Casanovas, J. y otros cuatro autores, La modelización molecular como herramienta para el diseño de nuevos polímeros conductores, Polímeros: Ciência e Tecnologia: (004), 239-244 (2005).

Choi, J., B. Lee y J.H. Kim, Synthesis and electroluminescent properties of m-conjugated copolymer based on 10-hexylphenothiazine and aromatic 1,2,4-triazole, Synthetic Met: 159(19-20), 1922-1927 (2009).

Curcó, D. y C. Alemán, Computational tool to model the packing of polycyclic chains: Structural analysis of amorphous polythiophene, J. Comput. Chem: 28(10), 1743-1749 (2007).

Derosa, P.A., A combined semiempirical-DFT study of oligomers within the finite-chain approximation, evolution from oligomers to polymers, J. Comput. Chem: 30(8), 1220-1228 (2009).

Haye, S., V.I. Slaveykova y J. Payet, Terrestrial ecotoxicity and effect factors of metals in life cycle assessment (LCA), Chemosphere: 68(8), 1489-1496 (2007).

Heeger, A. J., Semiconducting and Metallic Polymers: The Fourth Generation of Polymeric Materials (Nobel Lecture), Ang. Chem. Int. Ed: 40(14), 2591-2611 (2001). 
Hosoya, H., M. Aida, R. Kumagai y K. Watanabe, Analysis of the $\pi$-electronic structure of infinitely large networks. I. Some remarks on the characteristic polynomial and density of states of large polycyclic aromatic hydrocarbons, J. Comput. Chem: 8(4), 358-366 (1987).

Hughes, G. y M.R. Bryce, Electron-transporting materials for organic electroluminescent and electrophosphorescent devices, J. Mater. Chem: 15(1), 94-107 (2005).

Janietz, S., J. Barche, A. Wedel y D. Sainova, Poly(4-hexyl-1,2,4-triazole-4H): An Electron Semiconducting Analogue to Regioregular Poly(hexylthiophene), Macromol. Chem. Physic: 205(14), 1916-1922 (2004).

Jansson, E., J.P. Chandra y H. Ågren, Density functional study of triazole and thiadiazole systems as electron transporting materials, Chem. Phys: 330(1-2), 166-171(2006).

Kertesz, M., S. Yang y Y. Tian, Energy Gaps and their Control in Thiophene-Based Polymers and Oligomers. In: Handbook of Thiophene-Based Materials (I. F. Perepichka y D. F. Perepichka, eds.), 341-364, John Wiley \& Sons, Ltda (2009).

Kido, J., K. Hongawa, K. Okuyama y K. Nagai, Bright blue electroluminescence from poly(N-vinylcarbazole), App. Phys. Lett: 63(19), 2627-2629 (1993).

$\mathrm{Li}, \mathrm{A}, \mathrm{T}$. Yan y P. Shen, Exploring proton transfer in 1,2,3-triazole-triazolium dimer with ab initio method, J. Power Sources: 196(3), 905-910 (2011).

Liu, N., y otros siete autores, The enhanced electron injection by fluorinated tris-(8-hydroxy-quinolinato) aluminum derivatives in high efficient Si-anode OLEDs, J. Lumin: 131(2), 199-205 (2011).

Liu, Y., Y. Liu, y X. Zhan, High-Mobility Conjugated Polymers Based on Fused-Thiophene Building Blocks, Macromol. Chem. Physic: 212(5), 428-443 (2011).

MacDiarmid, A. G. y otros seis autores, The Concept of Doping of Conducting Polymers: The Role of Reduction Potentials, Phil. Trans. R. Soc. Lond A: 314(1528), 3-15 (1985).

MacDiarmid, A.G., Synthetic Metals: A Novel Role for Organic Polymers (Nobel Lecture), Angew. Chem. Int. Edit: 40(14), 2581-2590 (2001).

Myers, J. D. y J. Xue, Organic Semiconductors and their Applications in Photovoltaic Devices, Polym. Rev: 52(1), 1-37 (2012).

Nahmani, J., M.E. Hodson y S. Black, Effects of metals on life cycle parameters of the earthworm Eisenia fetida exposed to field-contaminated, metal-polluted soils, Environmental Pollution: 149(1), 44-58 (2007).

Nazare, A. N., S.N. Asthana, y H. Singh, Glycidyl azide polymer (GAP) an energetic component of advanced solid rocket propellants a review, J. Energ. Mater: 10(1), 43-63 (1992).

Raos, G, Computational reinvestigation of the bithiophene torsion potential, Chem. Phys. Lett: 379(3-4), 364-372 (2003).

Ripoll, J. D., A. Serna, D. Guerra y A. Restrepo, Electronic Structure Calculations on Helical Conducting Polymers, J. Phys. Chem. A: 114(41), 10917-10921 (2010).

Risko, C., E. Zojer, P. Brocorens, S.R. Marder y J. Brédas, Bis-aryl substituted dioxaborines as electrontransport materials: a comparative density functional theory investigation with oxadiazoles and siloles, Chem. Phys: 313(1-3), 151-157 (2005).

Rodríguez-Ropero, F., J. Casanovas y C. Alemán, Ab initio calculations on m-stacked thiophene dimer, trimer, and tetramer: Structure, interaction energy, cooperative effects, and intermolecular electronic parameters, J. Comput. Chem: 29(1), 69-78 (2008).

Salmón, M., K.K. Kanazawa, A.F. Diaz y M.A. Krounbi, Chemical route to pyrrole polymer films, J. Polym. Sci. Poly. Lett. Ed: 20(3), 187-193 (1982). 
Satyanarayana, K. C., J. Abildskov, R. Gani, G. Tsolou y V.G. Mavrantzas, Computer aided polymer design using multi-scale modelling, Braz. J. Chem. Eng: 27(3), 369-380 (2010).

Shaktawat, V, N. Jain, R. Saxena, N.S. Saxena y T.P. Sharma, Electrical conductivity and optical band gap studies of polypyrrole doped with different acids, J. Optoelectrons. Adv. Mat: 9(7), 2130-2132 (2007).

Shin, J.A., Y.G. Lim y K. Lee, Synthesis of Polymers Including Both Triazole and Tetrazole by Click Reaction, Bull. Korean. Chem. Soc: 32(2), 547-552 (2011).

Shirakawa, H.,y otros cuatro autores, Synthesis of electrically conducting organic polymers: halogen derivatives of polyacetylene, (CH)x, J. Chem. Soc. Chem. Commun: (16), 578-580 (1977).

Vivas-Reyes, R., L.D. Mercado, J. Anaya-Gil, A.G. Marrugo y E. Martínez, Theoretical study to evaluate polyfuran electrical conductivity and methylamine, methoxy substituent effects, J. Mol. Struc-Theochem: 861(1-3), 137-141 (2008).

Vivas-Reyes, R., F. Nuñez-Zarur y E. Martínez, Electronic structure and reactivity analysis for a set of Znchelates with substituted 8-hydroxyquinoline ligands and their application in OLED, Org. Electron: 9, 625634 (2008).

Wang, J.F. y otros seis autores, Theoretical Studies of the Absorption and Emission Properties of the Fluorene-Based Conjugated Polymers, Macromolecules: 37(9), 3451-3458 (2004).

Wang, T., S. Li, B. Yang, C. Huang y Y. Li, Thermal Decomposition of Glycidyl Azide Polymer Studied by Synchrotron Photoionization Mass Spectrometry, J. Phys. Chem. B: 111(10), 2449-2455 (2007).

Zhu, Y, I. Heim y B. Tieke, Red Emitting Diphenylpyrrolopyrrole (DPP)-Based Polymers Prepared by Stille and Heck Coupling, Macromol. Chem. Physic: 207(23), 2206-2214 (2006).

Zade, S.S y M. Bendikov, From oligomers to polymer: convergence in the HOMO-LUMO gaps of conjugated oligomers, Organic letters: 8(23), 5243-6 (2006). 
